\title{
Aberration of epidermal growth factor receptor expression in bone and soft-tissue tumors: protein overexpression, gene amplification and activation of downstream molecules
}

\author{
Yoh Dobashi, Nobuko Takei, Shioto Suzuki, Hiroko Yoneyama, Mitsuhiko Hanawa and \\ Akishi Ooi \\ Department of Pathology, Interdisciplinary Graduate School of Medicine and Engineering, University of \\ Yamanashi, Yamanashi, Japan
}

\begin{abstract}
In order to evaluate the involvement of epidermal growth factor receptor, and to analyze the correlation between gene aberration and protein expression in mesenchymal tumors, we examined protein expression by immunohistochemistry in $\mathbf{1 2 5}$ cases of bone and soft-tissue tumors. Furthermore, amplification of epidermal growth factor receptor gene was determined by fluorescence in situ hybridization. Positive immunostaining was found in 23 cases (18.4\%). Among these 23 cases, one of malignant fibrous histiocytoma showed the highest degree $(3+)$ of protein overexpression and gene amplification as clusters of hybridization signals, indicating homogeneously staining regions. The second case of malignant fibrous histiocytoma also showed a higher degree $(2+)$ of overexpression and coamplification of the epidermal growth factor receptor gene with the centromeric regions, indicating polysomy of chromosome 7. The levels of expression observed in immunohistochemistry were confirmed by immunoblotting and found to be comparable. Moreover, although expression of phosphorylated epidermal growth factor receptor was detected in those two cases of malignant fibrous histiocytoma, constitutive activation of extracellular signal-related protein kinase $1 / 2$ was not observed, suggesting that activation of epidermal growth factor receptor does not necessarily and constantly lead to signal transduction to the downstream molecules. In the remaining 123 cases, including 21 cases exhibiting weak $(1+)$ immunoreactivity, no gene amplification nor polysomy was found. Collectively, expression of epidermal growth factor receptor was observed not infrequently in mesenchymal tumors, but 'overexpression' is rare and can be attributed to an increase in gene copy number, resulting from amplification or polysomy. Although cases that scored positive for protein expression and/or gene amplification could be qualified candidates for antiepidermal growth factor receptor therapies, further examination of the status of downstream molecules in the signal cascade, such as phosphorylated epidermal growth factor receptor and extracellular signal-related protein kinase $1 / 2$, may be required as the process of therapeutic strategy.
\end{abstract}

Modern Pathology (2004) 17, 1497-1505, advance online publication, 9 July 2004; doi:10.1038/modpathol.3800218

Keywords: bone and soft-tissue tumors; epidermal growth factor receptor; extracellular signal-related protein kinase 1/2; fluorescence in situ hybridization; gene amplification; homogeneous staining region; polysomy

The epidermal growth factor receptor gene (EGFR), located on chromosome $7 \mathrm{p} 12$, is a proto-oncogene that encodes a membrane glycoprotein of $170 \mathrm{kDa}$ type I receptor tyrosine kinases (RTK). ${ }^{1,2}$ These RTKs have recently attracted much attention due to the development of chemotherapeutic agents

Correspondence: Dr Y Dobashi, MD, PhD, Department of Pathology, Faculty of Medicine, University of Yamanashi, 1110 Shimokato, Tamaho, Nakakoma, Yamanashi 409-3898, Japan.

E-mail: ydobashi@yamanashi.ac.jp

Received 20 April 2004; revised and accepted 7 June 2004; published online 9 July 2004 targeting them. Antibodies directed against the external domains of these receptors, or inhibitors of their intrinsic tyrosine kinase activities have been developed, and some have already been approved for clinical use in the patient of particular kinds of cancers. The humanized monoclonal antibody against HER-2 (Trastuzumab) for breast carcinoma is the most successful example. ${ }^{3}$ The treatment with this reagent has been demonstrated to extend median survival time in metastatic breast cancer patients exhibiting HER-2 overexpression, which is detected by immunohistochemistry and/or gene amplification confirmed by fluorescence in situ 
hybridization (FISH) analysis. ${ }^{4}$ With regards to EGFR, many types of epithelial malignancies have been reported to overexpress EGFR with or without corresponding gene amplification. ${ }^{5-7}$ Based on these facts, IMC-C225 (cetuximab), a monoclonal antibody targeting EGFR, was approved for colon cancers by the Food and Drug Administration in the United States in February 2004. In addition, ZD1839 (gefitinib), an inhibitor of tyrosine kinases of EGFR, has been used on patients of non-small-cell lung carcinomas. $^{8}$

Great effort has been made over the past few decades to improve the therapeutic strategy for malignant bone and soft-tissue tumors, which are often associated with unfavorable outcome. ${ }^{9}$ Consequently, there has been a large number of studies examining the overexpression of growth factors and their receptors, particularly of EGFR, in these tumors. ${ }^{10-12}$ Past studies have reported overexpression of EGFR in $81 \%$ of osteosarcoma, ${ }^{11} 67 \%$ of synovial sarcoma and in malignant peripheral nerve sheath tumor (MPNST), rhabdomyosarcoma and others at lower frequencies as well. ${ }^{10,12,13}$ However, only a few of these previous reports demonstrated clear membrane staining by immunohistochemistry, and gene amplification has been rarely documented. Accordingly, chemotherapy targeting EGFR has not been explored in this field of tumors.

We have previously examined overexpression of the EGFR protein and amplification of EGFR gene using immunohistochemistry and FISH, respectively, in gastric and colon cancers. Overall results demonstrated that high level of overexpression was found, up to $7.8 \%$ of the cases and that this overexpression was predominantly attributable to gene amplification. ${ }^{7,14}$ Furthermore, we also established definitive criteria on immunohistochemistry by those studies: the majority of 'strongly positive' $(2+$ to $3+)$ cases were associated with gene amplification whereas 'negative' or 'weakly positive' $(0$ to $1+)$ cases were never associated with amplification.
In this study, we examined bone and soft-tissue tumors for aberrations in EGFR expression and gene copy number as well as abnormal activation of the downstream molecule, extracellular signal-related protein kinase 1/2 (ERK1/2), in order to provide a rationale for the introduction of new adjuvant therapies for the patients with bone and soft-tissue tumors. Specifically, we tried (i) to determine the exact frequencies of EGFR overexpression; (ii) to clarify the relationship between protein overexpression and gene amplification of EGFR and (iii) to examine the possible relationship between expression of EGFR and phosphorylation of EGFR itself and ERK1/2 as a representative molecule downstream of EGFR-mediated signaling.

\section{Materials and methods}

\section{Tissue Samples}

For this study, 125 cases of bone and soft-tissue tumor (105 cases of malignant and 20 of benign tumors) were retrieved from the file of consecutive surgeries performed at the Departments of Surgery, and Orthopedic Surgery, University of Yamanashi Hospital between 1985 and 2003 as well as from the consultation files (Table 1). Histological diagnosis was made according to The World Health Organization Classifications of the Second Edition since nomenclature in the Third Edition is not prevalent. ${ }^{15}$

\section{Immunohistochemistry}

All tissue samples were routinely processed and serial sections were used for hematoxylin-eosin staining, immunohistochemistry and FISH analysis. IHC detection of EGFR protein was performed with an antibody against the external domain of EGFR (clone 113, Novocastra, Newcastle, UK; 1:40 dilution) following high-temperature antigen

Table 1 Histological types and immunohistochemical findings of 125 cases

\begin{tabular}{|c|c|c|c|c|c|c|c|c|}
\hline Histological type & - & Total & Histological type & $3+$ & $2+$ & $1+$ & - & Total \\
\hline Benign & & & Malignant & & & & & \\
\hline GCT & 2 & 2 & MFH & 1 & 1 & 3 & 30 & 35 \\
\hline Osteochondroma & 2 & 2 & Osteosarcoma & 0 & 0 & 8 & 12 & 20 \\
\hline Chondroma & 2 & 2 & Synovial sarcoma & 0 & 0 & 6 & 5 & 11 \\
\hline Dermatofibroma & 2 & 2 & Rhabdomyosarcoma & 0 & 0 & 2 & 5 & 7 \\
\hline Lipoma & 2 & 2 & Leiomyosarcoma & 0 & 0 & 1 & 12 & 13 \\
\hline Schwannoma & 2 & 2 & Liposarcoma & 0 & 0 & 1 & 4 & 5 \\
\hline Leiomyoma & 2 & 2 & Chondrosarcoma & 0 & 0 & 0 & 5 & 5 \\
\hline Osteoblastoma & 2 & 2 & Dermatofibrosarcoma protuberans & 0 & 0 & 0 & 3 & 3 \\
\hline Aneurysmal bone cyst & 1 & 1 & Fibrosarcoma & 0 & 0 & 0 & 2 & 2 \\
\hline Fibrous dysplasia & 1 & 1 & Chordoma & 0 & 0 & 0 & 2 & 2 \\
\hline Hemangioma & 1 & 1 & MPNST & 0 & 0 & 0 & 2 & 2 \\
\hline Chondroblastoma & 1 & 1 & & & & & & \\
\hline Total & 20 & 20 & Total & 1 & 1 & 21 & 82 & 105 \\
\hline
\end{tabular}

GCT, giant cell tumor; MFH, malignant fibrous histiocytoma; MPNST, malignant peripheral nerve sheath tumor. 
retrieval. ${ }^{7,14}$ Positively stained tumors were confirmed using an antibody against the cytoplasmic domain of EGFR (clone 25, Novocastra, 1:100). The specificity and sensitivity of these antibodies were previously confirmed., ${ }^{7,14}$

EGFR positivity in immunohistochemistry were evaluated by three of us (YD, NT, AO), who were unaware of the gene amplification. The intensity of EGFR immunoreactivity was scored using a four-tier system that we previously established as follows: ${ }^{7,14,16}$ negative, no discernible staining; $1+$, definite cytoplasmic staining and/or equivocal discontinuous membrane staining; $2+$, unequivocal membrane staining with moderate intensity; $3+$, strong and complete membrane staining. Samples exhibiting $1+, 2+$ or $3+$ staining were considered 'positive for expression', and $2+$ or $3+$ staining were defined as 'overexpression' since we have previously noted that only the cases showing $2+$ or $3+$ positivity in immunohistochemistry by our criteria were frequently associated with gene amplification of EGFR and HER2.. ${ }^{7,14,16}$

\section{FISH Analysis}

FISH was performed for all tumors showing a positive reaction of $1+$ to $3+$ in immunohistochemistry as well as five cases each of 'negative' scoring osteosarcoma and malignant fibrous histiocytoma. Gene amplification was determined using fluorescently labeled DNA probe sets (LSI EGFR/ CEP 7TM, Vysis, Downers Grove, IL, USA) containing a SpectrumOrangeTM-labelled EGFR-specific probe and a SpectrumGreenTM-labelled centromeric probe hybridized to centromeric region of the chromosome as the control to normalize copy number for the chromosome 7. FISH was performed using standard method with a modified intermittent, short-term microwave treatment during the initial period of hybridization. ${ }^{17}$ In short, after denaturing DNA, the sample slides in the hybridization mixture were placed in a microwave processor, irradiated for $3 \mathrm{~s}$ with 2-s intervals $(2.45 \mathrm{GHz}$, $300 \mathrm{~W}$ ), under constant temperature at $42^{\circ} \mathrm{C}$, and hybridized overnight. ${ }^{7,14,17}$ The sections were counterstained with $4^{\prime}, 6$-diamidine-2'-phenylindole dihydrochloride and $p$-phenylenediamine (DAPI II) (Vysis) and examined by a fluorescence microscope (Olympus, Tokyo, Japan) with a Triple Bandpass Filter (Vysis).

Chromosomal aberration was defined as follows: (i) a definite cluster or more than 10 EGFR signals were scored as 'amplification', (ii) more than two EGFR signals accompanied by centromere signals in more than $50 \%$ of the tumor cells were scored as 'polysomy', (iii) more than two signals of EGFR and of centromere in $20-50 \%$ of the tumor cells were scored as 'minimal aberration' and (iv) only two EGFR signals with centromere signals in more than
$80 \%$ of the tumor cells were scored as 'no significant abnormality'.,16

\section{Cell Lines and Tumor Tissues}

A431 (vulval squamous cell carcinoma) and Saos-2 cells (osteosarcoma) were available from RIKEN cell bank (Tukuba, Japan), and HOS cells (osteosarcoma) were from American Tissue Culture Collection (Manassas, VA, USA). A431 cells display amplification of the EGFR gene and protein overexpression. ${ }^{18}$ HOS cells also express EGFR protein. ${ }^{19}$ These cells were grown in RPMI supplemented with $10 \%$ fetal bovine serum.

Fresh fragments of tumor tissues were obtained from surgically resected specimens. These consisted of nine cases of malignant bone and soft-tissue tumor of which histopathological and immunohistochemical profiles are listed in Table 2.

\section{Immunoblotting Analysis}

Protein was extracted from fresh tissues and cultured cells by homogenizing in high-salt lysis buffer (0.5\% Nonidet P-40 (NP-40), $0.15 \%$ SDS, $50 \mathrm{mM}$ Tris-HCl (pH 8.0), 0.25 M NaCl, 5 mM EDTA, $50 \mathrm{mM}$ $\mathrm{NaF}, \quad 0.5 \mathrm{mM}$ phenylmethylsulfonyl fluoride (PMSF), $5 \mu \mathrm{g} / \mathrm{ml}$ of aprotinin, $5 \mu \mathrm{g} / \mathrm{ml}$ of leupeptin) supplemented with $1 \mathrm{mM} \mathrm{Na}_{3} \mathrm{VO}_{4}{ }^{20}{ }^{20}$ Equal amounts $(40 \mu \mathrm{g})$ were applied for the immunoblotting analysis. Primary antibodies were used at the following dilutions: anti-EGFR (same antibody used for IHC), 1:100; anti-phospho-EGFR (p-EGFR, \#2234, Cell Signaling, Beverly, MA, USA), 1:500; and antiphospho-ERK (p-ERK1/2, \#9106, Cell Signaling), 1:1000. Colorization was performed with nitroblue tetrazolium (NBT) and 5-bromo-4-chloro-3-indolphosphate (BCIP) (Promega, Madison, WI, USA). ${ }^{21}$

\section{Results}

\section{Immunohistochemistry}

EGFR was found to be expressed in 23 cases of malignant tumors $(18.4 \%$ of total, $21.9 \%$ of

Table 2 Profiles of the cases used for immunoblotting analysis

\begin{tabular}{llc}
\hline Sample number & Histological type & IHC \\
\hline 1 & MFH & $3+$ \\
2 & MFH & $2+$ \\
3 & MFH & $1+$ \\
4 & MFH & 0 \\
5 & Osteosarcoma & $1+$ \\
6 & Osteosarcoma & $1+$ \\
7 & Liposarcoma & $1+$ \\
8 & Synovial sarcoma & $1+$ \\
9 & Synovial sarcoma & 0
\end{tabular}

MFH, malignant fibrous histiocytoma; IHC, results of immunohistochemistry. 

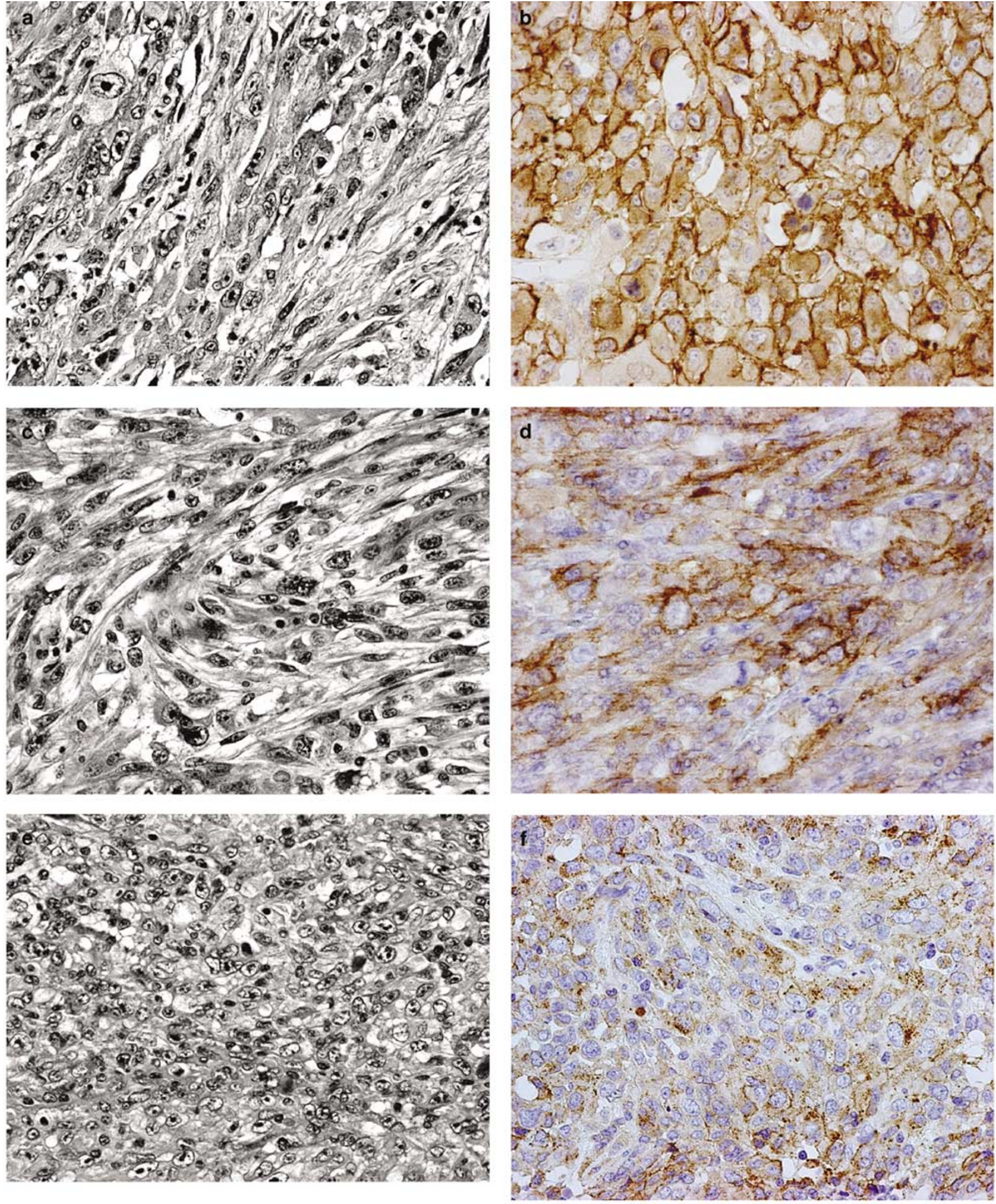

Figure 1 The results of immunohistochemical and FISH analyses in representative three cases of malignant fibrous histiocytoma. Hematoxylin-eosin stains (a, c, e), anti-EGFR stains (b, d, f) and Dual-color FISH of EGFR/centromere 7 (g, h, i) for Case 1 (a, b, g), Case 2 $(\mathbf{c}, \mathbf{d}, \mathbf{h})$ and Case 3(e, f,i) are shown. (b) Plasma membrane of tumor cells exhibiting $3+$ staining (Case 1). (d) Tumor cells overexpressing EGFR $(2+)$ occupied a limited area of the tumor (Case 2). (f) Faint membrane and cytoplasmic EGFR staining (1+) was observed (Case 3). (g) Tumor cells with large clustered signals of EGFR (Orange) were observed. Fibroblasts exhibiting two EGFR signals (disomy pattern) are seen among large tumor cells. (h) Coamplified EGFR signals (orange) accompanied by the identical numbers of centromere seven signals (green) are found in the nuclei. (i) Tumor cell nuclei exhibited predominantly disomy pattern with occasional increases of EGFR signals (orange), but no definitive cluster or signal increase in all cells. 
malignancy) of the 125 cases (Table 1): $3+$, one case (Case 1) of malignant fibrous histiocytoma; $2+$, one (Case 2) of malignant fibrous histiocytoma; $1+$, eight of osteosarcoma, six of synovial sarcoma, three of malignant fibrous histiocytoma, two of rhabdomyosarcoma and one of liposarcoma and leiomyosarcoma. EGFR expression was not detected in any of 20 benign tumors. In two cases evaluated as 'overexpression' $(3+$ and $2+)$, both were pleomorphic-storiform type of malignant fibrous histiocytoma. In Case 1 of malignant fibrous histiocytoma, more than $90 \%$ of tumor cells in the primary site were diffusely stained (Figure $1 \mathrm{a}$ and $\mathrm{b}$ ). In addition, the metastatic foci in the lymph node also showed $3+$ staining, but with a heterogeneous pattern admixed with unstained cells. Case 2 of malignant fibrous histiocytoma showed geographical staining pattern of $2+$ positivity, in $60 \%$ of the tumor cells (Figure 1c and d). No histological differences were noted between positive and negative areas within Case 2 or among positive and negative cases of malignant fibrous histiocytoma. Of the cases showing $1+$ staining, positively stained cells observed comprised less than $30 \%$ of the total tumor cells (Figure 1e and f). Among the 21 cases exhibiting $1+$ staining of EGFR in the primary tumors, a metastatic tumor in the lung was available in only one case (Case 4, malignant fibrous histiocytoma), which developed 3 years after the resection of the primary tumor, and it showed negative staining. All five cases of malignant fibrous histiocytoma that showed positive staining $(1+$ to $3+)$ were confirmed to have occurred in the soft tissue, such as within the thigh, buttock, retroperitoneum, arm and the shoulder (Table 3).

\section{FISH Analysis}

By FISH analysis, EGFR amplification was demonstrated in Case 1 of malignant fibrous histiocytoma ( $3+$ positivity in immunohistochemistry). The amplification pattern consisted of one or two distinct clusters with occasional scattered EGFR signals, and fewer than five centromere signals were observed (cluster type; Figure 1g and Table 3). This patient developed lymph node metastasis, which revealed EGFR-overexpressing cells $(3+)$, with amplification of the EGFR as observed in the primary tumor. In Case 2 of malignant fibrous
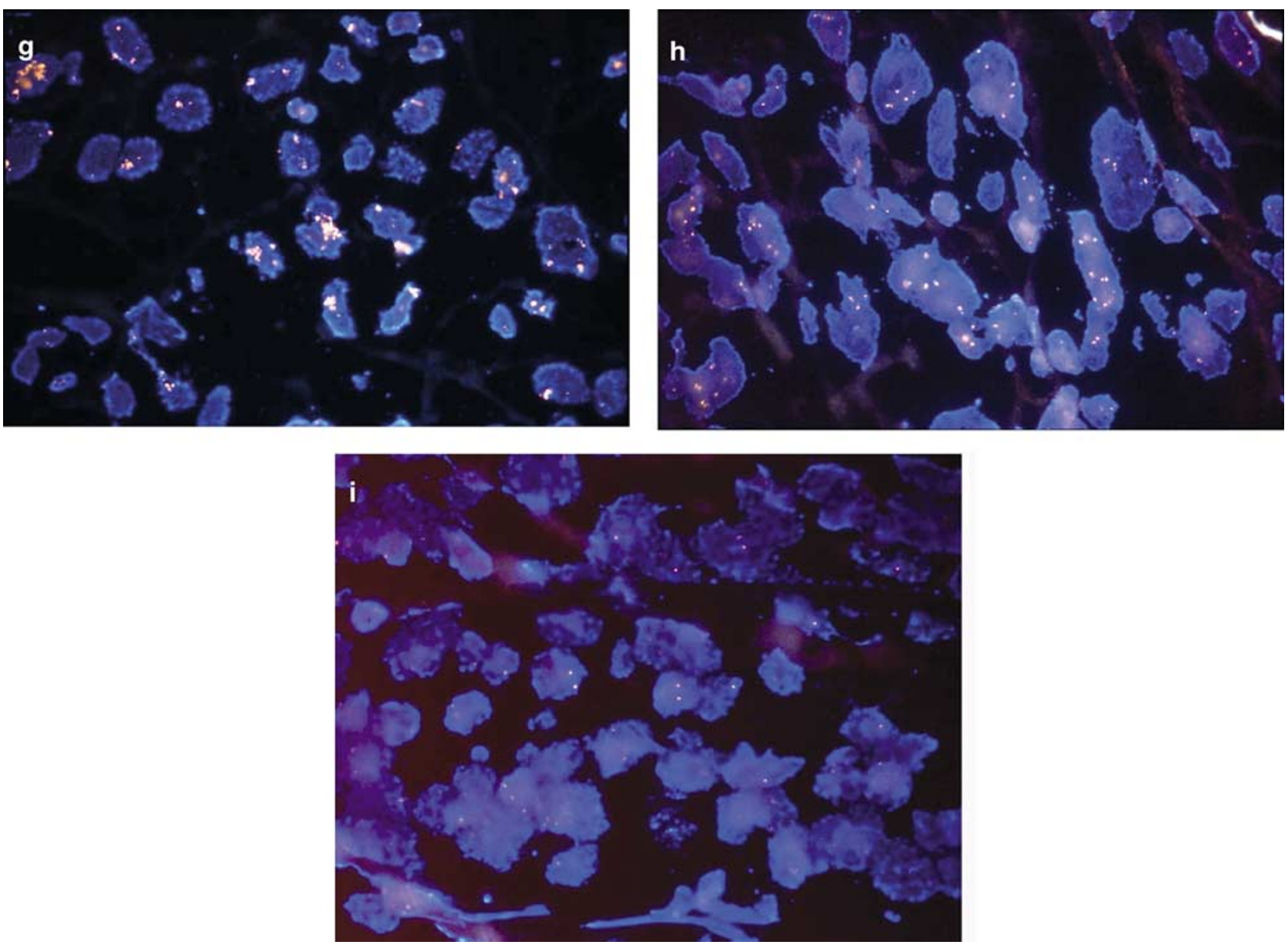

Figure 1 Continued. 
Table 3 Results of FISH analysis in IHC-positive cases

\begin{tabular}{llll}
\hline $\begin{array}{l}\text { Case } \\
\text { number }\end{array}$ & Histological type & IHC & $\begin{array}{l}\text { FISH type of } \\
\text { amplification }\end{array}$ \\
\hline 1 & MFH (right thigh) & $3+$ & LC+SS \\
$1^{\prime}$ & Metastatic lymph node & $3+$ & LC+SS \\
2 & MFH (left buttock) & $2+$ & polysomy \\
$2^{\prime}$ & Metastatic lymph node & $2+$ & - \\
3 & MFH (retroperitoneum) & $1+$ & - \\
4 & MFH (right arm) & $1+$ & MA \\
$4^{\prime}$ & Lung metastasis & - & - \\
5 & MFH (left shoulder) & $1+$ & - \\
6 & Osteosarcoma & $1+$ & MA \\
7 & Osteosarcoma & $1+$ & MA \\
8 & Osteosarcoma & $1+$ & MA \\
9 & Osteosarcoma & $1+$ & - \\
10 & Osteosarcoma & $1+$ & - \\
11 & Osteosarcoma & $1+$ & - \\
12 & Osteosarcoma & $1+$ & - \\
13 & Osteosarcoma & $1+$ & - \\
14 & Synovial sarcoma & $1+$ & MA \\
15 & Synovial sarcoma & $1+$ & MA \\
16 & Synovial sarcoma & $1+$ & - \\
17 & Synovial sarcoma & $1+$ & - \\
18 & Synovial sarcoma & $1+$ & - \\
19 & Synovial sarcoma & $1+$ & - \\
20 & Rhabdomyosarcoma & $1+$ & MA \\
21 & Rhabdomyosarcoma & $1+$ & - \\
22 & Liposarcoma & $1+$ & - \\
23 & Leiomyosarcoma & $1+$ & - \\
\hline
\end{tabular}

IHC, immunohistochemistry; MFH, malignant fibrous histiocytoma; LC, large clustered signal; SS, scattered signal.

${ }^{\mathrm{a}} \mathrm{MA}$, minimal aberration (more than two signals of EGFR and of centromere in $20-50 \%$ of tumor cells).

histiocytoma $(2+$ in immunohistochemistry), more than three, up to eight scattered EGFR signals were observed accompanied by the identical numbers of centromere signals ('polysomy'; Figure 1h). Metastatic lesions in the lymph node, showing positive reactivity of $2+$ in immunohistochemistry, also exhibited polysomy. Among the 21 cases of $1+$ staining, seven cases showed minimal aberration, that is, more than two signals of EGFR and centromere in tumor cells. However, these cells constituted less than $30 \%$ of the total tumor cells, which was not enough to be evaluated as polysomy by the definition used in this study. Moreover, in five (three of malignant fibrous histiocytoma and two cases of osteosarcoma) out of 10 cases (five cases each of malignant fibrous histiocytoma and osteosarcoma) selected as negative controls, a similar increase in the number of EGFR signals accompanied by the identical numbers of centromere signals was observed. No significant numerical aberration of the EGFR was observed in other cases exhibiting $1+$ staining or within the negative control cases (Figure 1i).

\section{Immunoblotting Analysis}

In order to confirm the results of immunohistochemistry and to examine the correlation between the expression levels of EGFR protein and gene copy number, we next performed EGFR-blotting on the collected tissue and cell samples. A431 cells exhibited the highest level of EGFR expression, depicted as a broad band around $170 \mathrm{kDa}$ (Figure 2). HOS cells also revealed detectable level of expression, whereas Saos-2 did not. EGFR expression level identified in Case 1 of malignant fibrous histiocytoma was much higher than that in HOS, albeit not as high as A431. Expression of EGFR in Case 2 of malignant fibrous histiocytoma was also detectable, but at a lower level than that of Case 1. In other sarcoma cases, both two cases of osteosarcoma (1+ staining) revealed low, but detectable levels of protein. All of these tissue samples contained a protein of $170 \mathrm{kDa}$, suggesting the absence of truncated protein. The other cases showing $1+$ staining as well as a negative case of synovial sarcoma, did not show detectable levels of EGFR.

We next investigated the phosphorylation of EGFR and its downstream signaling molecules using phosphorylated protein-specific antibodies. p-EGFR blotting of A431 revealed one broad band of the phosphorylated form of EGFR (Figure 2). Similarly, Case 1 of malignant fibrous histiocytoma also revealed lower, but detectable level of p-EGFR. Case 2 of malignant fibrous histiocytoma showed further lower level, but definitive expression was identified. p-EGFR was not detected in two cases of osteosarcoma and HOS cells, even though overall expression of EGFR in those cases was elevated.

Similarly, phosphorylation of ERK1/2 was investigated by p-ERK1/2 blotting. Although doublet bands of $44 / 42 \mathrm{kDa}$, corresponding to the phosphorylated forms of ERK1/2, were observed in all samples of tissue lysates and cell lines, the expression levels of EGFR and p-ERK1/2 did not correlate to one another (Figure 2). For instance, Case 1 of malignant fibrous histiocytoma, which had the
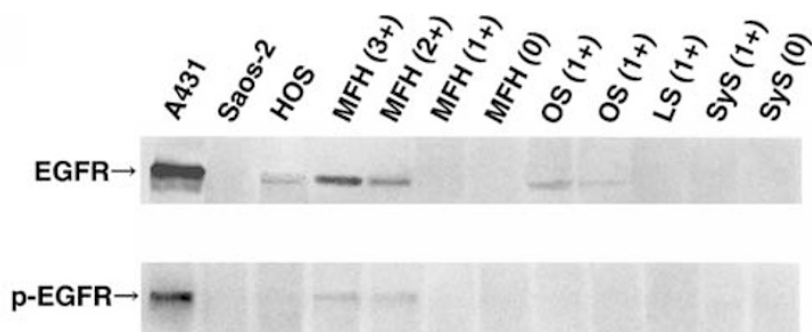

$\mathrm{p}-\mathrm{ERK} 1 / 2 \Rightarrow$

Figure 2 Protein levels of EGFR, phospho-EGFR (p-EGFR) and phospho-ERK1/2 (p-ERK1/2). Cell lysates $(40 \mu \mathrm{g})$ were subjected to immunoblotting analysis to detect EGFR, p-EGFR and p-ERK1/ 2. The positions of the EGFR and p-EGFR as well as those of p-ERK1/2 doublets are indicated by arrows. Abbreviations: EGFR, epidermal growth factor; p-EGFR, phosphorylated EGFR; p-ERK1/ 2, phosphorylated extracellular signal-related protein kinase 1/2; MFH, malignant fibrous histiocytoma; OS, osteosarcoma; LS, liposarcoma; SyS, synovial sarcoma. 
highest level of EGFR and p-EGFR next to A431, showed lower level of p-ERK1/2. This is compared to one of osteosarcoma where p-ERK1/2 was relatively abundant, although little to no EGFR and p-EGFR was detected.

\section{Discussion}

Despite remarkable advances in the therapy of human malignant tumors, particularly of bone and soft-tissue tumors, overall results are not satisfactory, and new strategies are required to improve treatment efficacy. Recent clinical studies have reported that that novel agents directed against EGFR show some benefits in a treatment-refractory cancer population. ${ }^{822,23}$ Moreover, it was demonstrated that the efficacy of chemotherapy or radiation therapy is enhanced by coadministration of cetuximab or gefitinib in those tumors exhibiting EGFR overexpression. ${ }^{24,25}$ We and others, in this line, have examined the incidence of EGFR overexpression in carcinomas of the lung, stomach and colon, and found that it ranged from 7.8 to $62 \%{ }^{2,5,7,26}$ However, further details regarding the relationship between the sensitivity to gefitinib and various pathobiological characteristics of tumors, such as histological type, EGFR expression level, and the presence/absence of gene amplification have not been available. Toward this end, we therefore planned this study to clarify these relationships, ultimately, in order to determine whether EGFR might be a suitable target of therapy, even for a minor population of bone and soft-tissue tumor cases.

EGFR has been frequently described to be 'expressed' or 'overexpressed' in cultured cells of human mesenchymal tumors, in particular, of osteosarcoma. ${ }^{27-31}$ Indeed, in the present study, we observed positive EGFR staining by immunohistochemistry in 23 out of 105 of sarcoma cases (21.9\%). However, previous reports described expression of EGFR in sarcomas with higher frequencies than those in the present study; $81 \%$ of osteosarcoma vs $40.0 \%$ in ours, $67 \%$ of synovial sarcoma vs $54.5 \%$ in ours, and $60 \%$ of MPNST vs $0 \%$ in ours. ${ }^{11-13}$ These discrepancies may arise from the differences in the criteria for evaluating the results of immunohistochemistry. For example, weak cytoplasmic staining was interpreted as ' 1 +' for 21 cases in this study, whereas these samples may have been scored higher in the previous reports. Thus, we examined protein overexpression by immunohistochemistry and immunoblotting and also performed FISH to further detail the expression profile of EGFR. In our FISH analysis, none of $1+$ cases exhibited gene amplification, and protein levels evaluated by immunoblotting were significantly lower than those in tumors where gene amplification or polysomy had occurred. As a result, we concluded that $2+$ and $3+$ positivity in immunohistochemistry should be de- signated as 'overexpression', distinct from the cases of $1+$ positivity, and this 'overexpression' of EGFR protein was the result of increased EGFR copy number due to gene amplification or polysomy.

Among the several methods, FISH analysis on paraffin-embedded tissues is the most reliable method to clarify gene amplification in human tumors since the precise copy numbers and the amplification types can be visualized and compared with protein expression in immunohistochemistry on a cell-by-cell basis. Highly amplified DNA is found either in the form of expanded chromosomal regions, homogeneously staining regions, or in double minute chromosomes (DM), which are centromere-free circular structures.,16,32 Clustered signals generally correspond to amplified signals in homogeneously staining regions, and scattered signals correspond to DM. ${ }^{32}$ In the present study, Case 1 of malignant fibrous histiocytoma showed amplification of homogeneously staining regiontype. In contrast, increase in both gene copy number and chromosome number is indicative of polysomy, which was observed in Case 2 of malignant fibrous histiocytoma. In addition to gene amplification, polysomy has recently been described to play a role in overexpression of EGFR in lung carcinomas and HER-2 in breast carcinoma. ${ }^{26,33}$ Our result may represent a similar phenomenon in the EGFR of bone and soft-tissue tumors. Minimal aberrations observed in several cases of ' $1+$ ' and 'negative' scoring probably originated in the complex and random increase of chromosomes in sarcoma cells.

Although previous study demonstrated EGFR gene amplification in $2 \%$ of sarcoma cases by Southern blotting analysis, histological subtype was not mentioned. ${ }^{10}$ Another group described EGFR amplification in one of malignant fibrous histiocytoma out of 20 cases of sarcomas. ${ }^{34}$ However, this latter study was based on immunohistochemical analysis utilizing an amplification/overexpression-specific antibody generated by the authors, and was not accompanied by a genetic analysis. Pathologists sometimes have a hard time to differentiate malignant fibrous histiocytoma from undifferentiated carcinoma when the tumor occurs in the organ with epithelial tissue. However, in this series, five cases of malignant fibrous histiocytoma that showed immunohistochemical positivity $(1+$ to $3+$ ) were confirmed to have occurred in the soft tissue and revealed no histological features of epithelial component. Thus, it seems unlikely that the cases of undifferentiated carcinoma were misdiagnosed as malignant fibrous histiocytoma.

To our knowledge, this is the first study to demonstrate EGFR amplification/polysomy in histologically affirmed cases.

It is apparent that, in addition to the expression of EGFR, understanding of the phosphorylation state of the molecules involved in the signaling cascade functioning downstream of EGFR would also greatly improve therapeutic strategies targeting EGFR. 
However, the precise mechanisms by which growth factor-induced cell surface signals are transmitted to the nucleus are not understood in human tumors. Thus, we examined the levels of p-EGFR and pERK1/2 in correlation with total amount of EGFR protein. A positive correlation was observed between the level of EGFR expression and p-EGFR signals in A431 cells and the two cases of malignant fibrous histiocytoma. However, this was not the case for p-ERK1/2, where expression of this phosphoprotein did not correlate with that of EGFR or pEGFR. The discrepancy between EGFR and p-ERK1/ 2 expression could be explained by one of the following: First, this may reflect a temporal profile in which ERK1/2 is activated within a short amount of time and constitutive activation is not a prerequisite for signal transduction. Second, an alternative EGFR-signal transduction pathways, such as JAK/STAT, is activated. ${ }^{35}$ Finally, signals by overexpressed EGFR may simultaneously lead to upregulation of cognate phosphatase activity, such as MAP kinase phosphatases (MKPs). ${ }^{36}$ Consistent with this idea, several lines of evidence have suggested that neither the expression level nor the phosphorylation status of EGFR seems to correlate with the sensitivity to gefitinib. ${ }^{24}$ If this is the case, anti-EGFR therapy against tumors overexpressing EGFR may be too simplistic.

The correlations between EGFR overexpression and patients' prognosis were contradictorily described in carcinomas of the various organs: it was found to be associated with shorter survival in carcinomas of the head and neck, and the ovary, yet no correlation in lung carcinoma. ${ }^{2,26}$ In this study, due to our limited sample size, the predictive power of this molecule could not be precisely evaluated.

In conclusion, anti-EGFR therapies may be appropriate for patients with mesenchymal tumors which score as 'positive' for EGFR expression. It should be noted, however, that tumors that overexpress EGFR due to gene amplification may require gene-targeting therapy rather than EGFR-protein inhibitor-based therapy, since expression levels in these cases are extraordinary higher than those in EGFR-expressing tumors without gene amplification. Accordingly, before proceeding to anti-EGFR therapy directly, it is advisable that the status of EGFR amplification should be precisely determined and that the correlation between EGFR overexpression and the activation of downstream molecules should be carefully evaluated in each case.

\section{Acknowledgements}

This work is supported by grant-in-aid for Scientific Research, No. C 14570161, No. C 15590298 and No. C 16590272, from the Japanese Ministry of Education, Culture, Sports, Science and Technology, in Japan and by the grant from The Yamanashi Academy of Sciences.

\section{References}

1 Todaro GJ, Rose TM, Spooner CE, et al. Cellular and viral ligands that interact with the EGF receptor. Semin Cancer Biol 1990;4:257-263.

2 Nicholson RI, Gee JM, Harper ME. EGFR and cancer prognosis. Eur J Cancer 2001;37:9-15.

3 Smith IE. New drugs for breast cancer. Lancet 2002; 360:790-792.

4 Treish I, Schwartz R, Lindley C. Pharmacology and therapeutic use of trastuzumab in breast cancer. Am J Health Syst Pharm 2000;22:2063-2076.

5 Sakai K, Mori S, Kawamoto T, et al. Expression of epidermal growth factor receptors on normal human gastric epithelia and gastric carcinomas. J Natl Cancer Inst 1986;77:1047-1052.

6 Dolf G, Meyn RE, Curley D, et al. Extrachromosomal amplification of the epidermal growth factor receptor gene in a human colon carcinoma cell line. Genes Chromosomes Cancer 1991;3:48-54.

7 Takehana T, Kunitomo K, Suzuki S, et al. Overexpression of epidermal growth factor receptor in gastric carcinomas is rare but occurs predominantly by gene amplification. Clin Gastroenterol Hepatol 2003;1:438-445.

8 Rusnak DW, Affleck K, Cockerill SG, et al. The characterization of novel, dual ErbB-2/EGFR, tyrosine kinase inhibitors: potential therapy for cancer. Cancer Res 2001;61:7196-7203.

9 Saeter G, Alvegard TA, Monge OR, et al. Ifosfamide and continuous infusion etoposide in advanced adult soft tissue sarcoma. A Scandinavian Sarcoma Group Phase II Study. Eur J Cancer 1997;33:1551-1558.

10 Duda RB, Cundiff D, August CZ, et al. Growth factor receptor and related oncogene determination in mesenchymal tumors. Cancer 1993;71:3526-3530.

11 Oda Y, Wehrmann B, Radig K, et al. Expression of growth factors and their receptors in human osteosarcomas. Immunohistochemical detection of epidermal growth factor, platelet-derived growth factor and their receptors: its correlation with proliferating activities and p53 expression. Gen Diagn Pathol 1995;141: 97-103.

12 Nielsen TO, Hsu FD, O’Connell JX, et al. Tissue microarray validation of epidermal growth factor receptor and SALL2 in synovial sarcoma with comparison to tumors of similar histology. Am J Pathol 2003;163:1449-1456.

13 Gusterson B, Cowley G, McIlhinney J, et al. Evidence for increased epidermal growth factor receptors in human sarcomas. Int J Cancer 1985;36:689-693.

14 Ooi A, Takehana T, Li XL, et al. Protein overexpression and gene amplification of HER-2 and epidermal growth factor receptor in colorectal cancers. An immunohistochemical and fluorescence in situ hybridization study. Mod Pathol, in press.

15 Fletcher CDM, Unni KK, Mertens F. World Health Organization Classification of Tumours. Pathology and Genetic of Tumours of Soft Tissue and Bone. IARC Press: Lyon, 2002.

16 Takehana T, Kunitomo K, Kono K, et al. Status of cerbB-2 in gastric adenocarcinoma: a comparative study of immunohistochemistry, fluorescence in situ hybridization and enzyme-linked immuno-sorbent assay. Int J Cancer 2002;98:833-837.

17 Kitayama Y, Igarashi H, Sugimura H. Initial intermittent microwave irradiation for fluorescence in situ 
hybridization analysis in paraffin-embedded tissue sections of gastrointestinal neoplasia. Lab Invest 2000;80:779-781.

18 Linsley PS, Fox CF. Direct linkage of EGF to its receptor: characterization and biological relevance. J Supramol Struct 1980;14:441-459.

19 Pinski J, Schally AV, Halmos G, et al. Somatostatin analog RC-160 inhibits the growth of human osteosarcomas in nude mice. Int J Cancer 1996;65:870-874.

20 Dobashi Y, Shoji M, Kitagawa M, et al. Simultaneous suppression of cdc2 and cdk2 activities induces neuronal differentiation of PC12 cells. J Biol Chem 2000;275:12572-12580.

21 Noguchi T, Dobashi Y, Minehara H, et al. Involvement of cyclins in cell proliferation and their clinical implications in soft tissue smooth muscle tumors. Am J Pathol 2000;156:2135-2147.

22 Bonner JA, Raisch KP, Trummell HQ, et al. Enhanced apoptosis with combination C225/radiation treatment serves as the impetus for clinical investigation in head and neck cancers. J Clin Oncol 2000;18:47-53.

23 Gibbs JB. Anticancer drug targets: growth factors and growth factor signaling. J Clin invest 2000;105:9-13.

24 Sirotnak FM, Zakowski MF, Miller VA, et al. Efficacy of cytotoxic agents against human tumor xenografts is markedly enhanced by coadministration of ZD1839 ('Iressa'), and inhibitor of EGFR tyrosine kinase. Clin Cancer Res 2000;6:4885-4892.

25 Baselga J, Norton L, Masui H, et al. Antitumor effect of doxorubicin in combination with anti-epidermal growth factor monoclonal antibodies. J Natl Cancer Inst 1993;85:1327-1333.

26 Hirsch FR, Varella-Garcia M, Bunn Jr PA, et al. Epidermal growth factor receptor in non-small-cell lung carcinomas: correlation between gene copy number and protein expression and impact on prognosis. J Clin Oncol 2003;21:3798-3807.

27 Witlox MA, Van Beusechem VW, Grill J, et al. Epidermal growth factor receptor targeting enhances adenoviral vector based suicide gene therapy of osteosarcoma. J Gene Med 2002;4:510-516.
28 Gerharz CD, Ramp U, Reinecke P, et al. Analysis of growth factor-dependent signalling in human epithelioid sarcoma cell lines. Clues to the role of autocrine, juxtacrine and paracrine interactions in epithelioid sarcoma. Eur J Cancer 2000;36: 1171-1179.

29 Beech D, Pollock RE, Tsan R, et al. Epidermal growth factor receptor and insulin-like growth factor-I receptor expression and function in human soft-tissue sarcoma cells. Int J Oncol 1998;12:329-336.

30 Fishman D, Galitzki L, Priel E, et al. Epidermal growth factor regulates protein kinase A activity in murine fibrosarcoma cells: differences between metastatic and nonmetastatic tumor cell variants. Cancer Res 1997, 57:5410-5415.

31 De Giovanni C, Landuzzi L, Frabetti F, et al. Antisense epidermal growth factor receptor transfection impairs the proliferative ability of human rhabdomyosarcoma cells. Cancer Res 1996;56:3898-3901.

32 Fried M, Feo S, Heard E. The role of inverted duplication in the generation of gene amplification in mammalian cells. Biochem Biophys Acta 1991 1090:143-155.

33 Lal P, Salazar PA, Ladanyi M, et al. Impact of polysomy 17 on HER-2/neu immunohistochemistry in breast carcinomas without HER-2/neu gene amplification. J Mol Diagn 2003;5:155-159.

34 Jungbluth AA, Stockert E, Huang HJ, et al. A monoclonal antibody recognizing human cancers with amplification/overexpression of the human epidermal growth factor receptor. Proc Natl Acad Sci USA 2003;100:639-644.

35 Grandis JR, Drenning SD, Zeng Q, et al. Constitutive activation of Stat3 signaling abrogates apoptosis in squamous cell carcinogenesis in vivo. Proc Natl Acad Sci USA 2000;97:4227-4232.

36 Bokemeyer D, Sorokin A, Yan M, et al. Induction of mitogen-activated protein kinase phosphatase 1 by the stress-activated protein kinase signaling pathway but not by extracellular signal-regulated kinase in fibroblasts. J Biol Chem 1996;271:639-642. 